\title{
Mid-infrared-perturbed molecular vibrational signatures in plasmonic nanocavities
}

\author{
Rohit Chikkaraddy $\mathbb{1}^{1 \times}$, Angelos Xomalis $\mathbb{E}^{1,2}$, Lukas A. Jakob and Jeremy J. Baumberg $\mathbb{D}^{1 凶}$
}

\begin{abstract}
Recent developments in surface-enhanced Raman scattering (SERS) enable observation of single-bond vibrations in real time at room temperature. By contrast, mid-infrared (MIR) vibrational spectroscopy is limited to inefficient slow detection. Here we develop a new method for MIR sensing using SERS. This method utilizes nanoparticle-on-foil (NPoF) nanocavities supporting both visible and MIR plasmonic hotspots in the same nanogap formed by a monolayer of molecules. Molecular SERS signals from individual NPOF nanocavities are modulated in the presence of MIR photons. The strength of this modulation depends on the MIR wavelength, and is maximized at the 6-12 $\mu \mathrm{m}$ absorption bands of $\mathrm{SiO}_{2}$ or polystyrene placed under the foil. Using a single-photon lock-in detection scheme we time-resolve the rise and decay of the signal in a few $100 \mathrm{~ns}$. Our observations reveal that the phonon resonances of $\mathrm{SiO}_{2}$ can trap intense MIR surface plasmons within the Reststrahlen band, tuning the visible-wavelength localized plasmons by reversibly perturbing the localized few-nm-thick water shell trapped in the nanostructure crevices. This suggests new ways to couple nanoscale bond vibrations for optomechanics, with potential to push detection limits down to single-photon and single-molecule regimes.
\end{abstract}

\section{Introduction}

Optical detection methods in the mid-infrared regime (MIR, 3-15 $\mu \mathrm{m}$ ) with single-photon sensitivity have wide implications in astrophysics and molecular nanoscience. Molecules and polar dielectric systems have characteristic bond vibrations and phonon modes across MIR wavelengths ${ }^{1-6}$. For ultrasmall sample volumes, optical detection (or pumping) of these modes gives low signals and is challenging due to the weak far-field coupling of these vibrations and low quantum efficiencies of MIR detectors $^{7,8}$. Fourier transform infrared spectroscopy methods with photoconductive detectors (MCT) remain the workhorse for MIR detection, but they are slow, often require cryogenic cooling, and cannot approach the quantum limit. Upconverting low-energy MIR photons to

\footnotetext{
Correspondence: Rohit Chikkaraddy (rc621@cam.ac.uk) or

Jeremy J. Baumberg (jjb12@cam.ac.uk)

${ }^{1}$ NanoPhotonics Centre, Cavendish Laboratory, Department of Physics, JJ Thompson Avenue, University of Cambridge, Cambridge CB3 OHE, UK. ${ }^{2}$ Present address: Empa, Swiss Federal Laboratories for Materials Science and Technology, Laboratory for Mechanics of Materials and Nanostructures, Thun, Switzerland
}

high-energy visible photons would significantly benefit from single-photon-sensitive semiconductor (CCD, CMOS) technologies ${ }^{9-14}$. However, the poor conversion efficiencies and small spatial overlap of MIR and visible photons pose significant challenges ${ }^{15}$.

Recent developments have circumvented the limitations associated with optical diffraction at long wavelengths by using near-field tip scanning (s-SNOM), photothermal infrared (PTIR) $)^{16,17}$ and far-field mid-infrared photothermal microscopy $(\mathrm{MIP})^{18}$. s-SNOM still relies on MCT-based detection schemes but can overcome diffraction limits from near-field scanning tips. Near-field (PTIR) and far-field (MIP) photothermal methods instead utilize efficient detection in the visible. The modulated MIR laser beam changes the reflection/transmission of a visible beam due to thermal expansion, pressure waves, refractive index changes or Grüneisen changes in the medium, which are efficiently detected through lock-in methods ${ }^{18}$. Even though the visible detectors used are fast and efficient, the signals obtained in PTIR and MIP are limited by thermal diffusivities on millisecond timescales.

\section{(c) The Author(s) 2022, corrected publication 2022}

(c) (i) Open Access This article is licensed under a Creative Commons Attribution 4.0 International License, which permits use, sharing, adaptation, distribution and reproduction cc) in any medium or format, as long as you give appropriate credit to the original author(s) and the source, provide a link to the Creative Commons license, and indicate if changes were made. The images or other third party material in this article are included in the article's Creative Commons license, unless indicated otherwise in a credit line to the material. If material is not included in the article's Creative Commons license and your intended use is not permitted by statutory regulation or exceeds the permitted use, you will need to obtain permission directly from the copyright holder. To view a copy of this license, visit http://creativecommons.org/licenses/by/4.0/. 
a

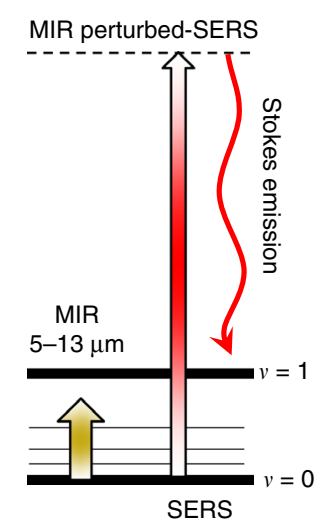

b

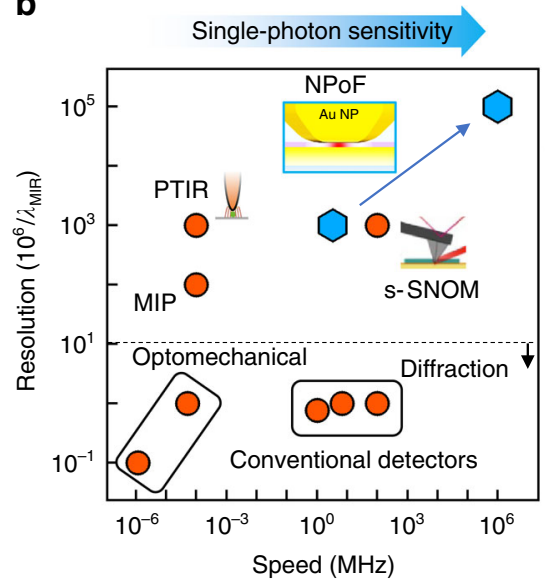

C

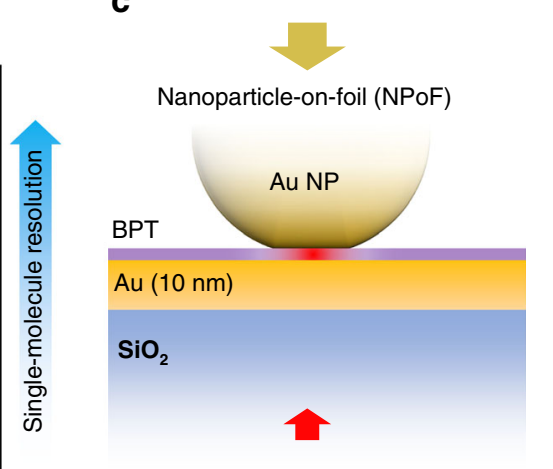

d

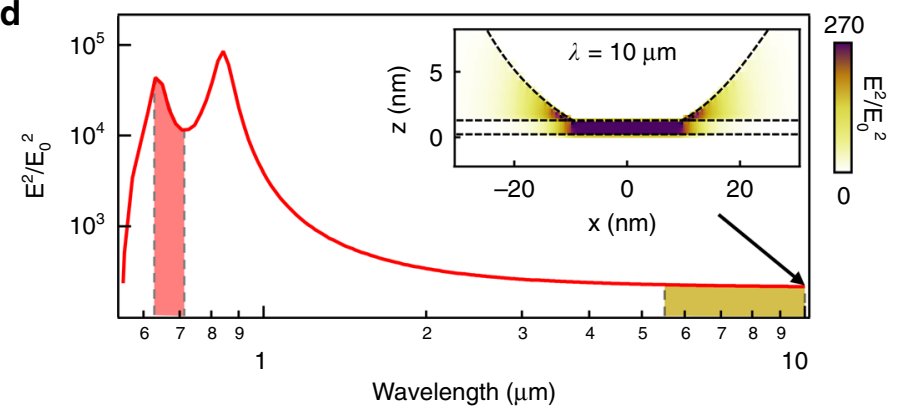

e

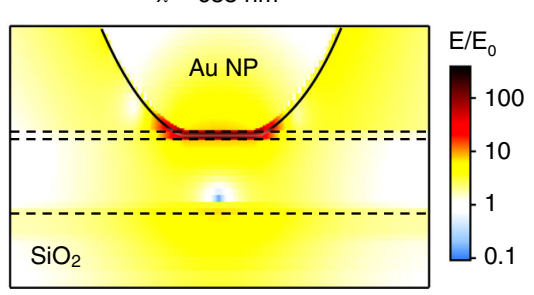

Fig. 1 Coupling MIR and visible light into plasmonic nanogaps. a Energy diagram of MIR-perturbed SERS from molecule with ground $(v=0)$ and excited $(v=1)$ vibrational states. $\mathbf{b}$ Landscape of MIR detection speed and resolution comparing conventional and state-of the-art detection schemes with the NPoF devices. Faster detectors can distinguish single MIR photon arrival times in the detection stream. c Nanoparticle-on-foil (NPoF) constructed on thin metal film. d Simulated electromagnetic field enhancement vs wavelength in the center of NPoF gap. Inset shows enhanced light intensity at MIR $\lambda=10 \mu \mathrm{m}$. Shaded regions show perturbed SERS (red) and MIR pumping (yellow). e Simulated electromagnetic field enhancement at $633 \mathrm{~nm}$ used for SERS measurements

These challenges can be addressed by upconversion which utilizes cavity optomechanical approaches for efficient MIR detection. When MIR light impinges on an optical resonator it can excite mechanical resonances which are read out optically, allowing measurement at room temperature with low noise ${ }^{19-22}$. Here the detection limits are set by optomechanical coupling strengths $(g)$, proportional to the quality factor of the mechanical mode. However, the diffraction-limited size of such cavities limits $g$ to less than $1 \mathrm{MHz}$ and thus functions worse than conventional MCT-based detectors. Intriguingly, the mechanical motion can now be replaced by vibrating bonds in molecules (Fig. 1a), opening clear avenues for molecular optomechanics and photochemistry ${ }^{23,24}$. This landscape of detection speed and resolution towards single-photon and single-molecule sensitivity shows how these diverse detection methods compare (Fig. 1b).

Here we develop a MIR-perturbed surface-enhanced Raman scattering (SERS) method which uses singlemolecule-sensitive metal nanocavities. The system is constructed using gold nanoparticles (AuNP) on a thin foil of planar Au with vibrating molecules assembled in the gap formed between them (Fig. 1c). The strong visible-light confinement in the nanogap provides enhanced $\left(>10^{9}\right)$ Raman scattering from the molecules in the gap, acting as a near-field probe. In this detection scheme, MIR light is absorbed in molecular bonds on the foil significantly altering the Stokes and anti-Stokes Raman signals at visible wavelengths, which can easily be detected (Fig. 1a). The interaction of light and matter in these sub-nm mode volumes allows extreme sensitivity to (in principle) single MIR photon with resolution down to a single molecule (Fig. 1b).

\section{Results}

Coherent electron oscillations coupled with light (plasmon polaritons) trap electromagnetic (EM) fields around metal nanostructures giving a resonant optical response in the visible and broad weaker optical response spanning from visible to MIR wavelengths ${ }^{25-30}$. While single metal nanoparticles do not provide sufficient field enhancement needed for robust single-molecule SERS, 
nanogap confinement improves this greatly. Here we exploit a multilayer nanoparticle-on-foil (NPoF) cavity that resonantly enhances the near-field at visible wavelengths in addition to giving a broad MIR optical response from lighting rod effects ${ }^{31,32}$. This structure consists of a faceted gold nanoparticle placed $\sim 1.3 \mathrm{~nm}$ above a thin $\mathrm{Au}$ film $(10 \mathrm{~nm})$ deposited on a $\mathrm{SiO}_{2}$ substrate (Fig. 1c). The gap distance between the AuNP and Au film is set by the monolayer height of biphenyl-4-thiol (BPT) molecules preassembled from solution onto the film before AuNP deposition. The resulting NPoF structure supports plasmonic $(l m)=(10)$ and $(20)$ cavity resonances at $850 \mathrm{~nm}$ and $650 \mathrm{~nm}$ with $E / E_{0}>100$ (Fig. 1e) ${ }^{33-35}$. This gives strong SERS and a broad uniform near-field enhancement across the MIR absorption wavelengths of 5-15 $\mu \mathrm{m}$ (Fig. 1d). The NPoF is designed for optimal spatial overlap of visible and MIR light which is vital for MIRperturbed SERS.

To study the MIR-perturbed SERS from these cavities, we direct a tunable MIR pump beam $(500 \mu \mathrm{W}$ average power) and $633 \mathrm{~nm}$ SERS probe $(150 \mu \mathrm{W}$ average power $)$ onto individual NPoF cavities (Fig. 2a). The $633 \mathrm{~nm}$ laser is focused through the $\mathrm{SiO}_{2}$ substrate while the MIR pump is focused via a Cassegrain objective from the airside, with estimated spot diameters on the sample of $1 \mu \mathrm{m}$ for $633 \mathrm{~nm}$ and $20 \mu \mathrm{m}$ for the MIR beam at $\lambda=10 \mu \mathrm{m}$ (Supporting Information, Fig. S1). Both beams are coaligned onto the sample and the back-scattered SERS from BPT molecules is collected through the $\mathrm{SiO}_{2}$ substrate and routed to the spectrometer. The NPoF supports a unique dual configuration with metal-insulator-metal (MIM) gap mode at the AuNP-foil junction coupling to the insulator-metal-insulator (IMI) mode at the air-foil-glass interface, resulting in tightly confined MIMI modes which radiate SERS light predominately into the glass medium ${ }^{36}$.

The NPoF cavities provide stable SERS signals upon laser illumination at $633 \mathrm{~nm}$, with characteristic BPT vibrational lines at $1080 \mathrm{~cm}^{-1}$ and $1585 \mathrm{~cm}^{-1}$ (Fig. 2b). ${ }^{37}$ The spectral intensity variation obtained from time-series
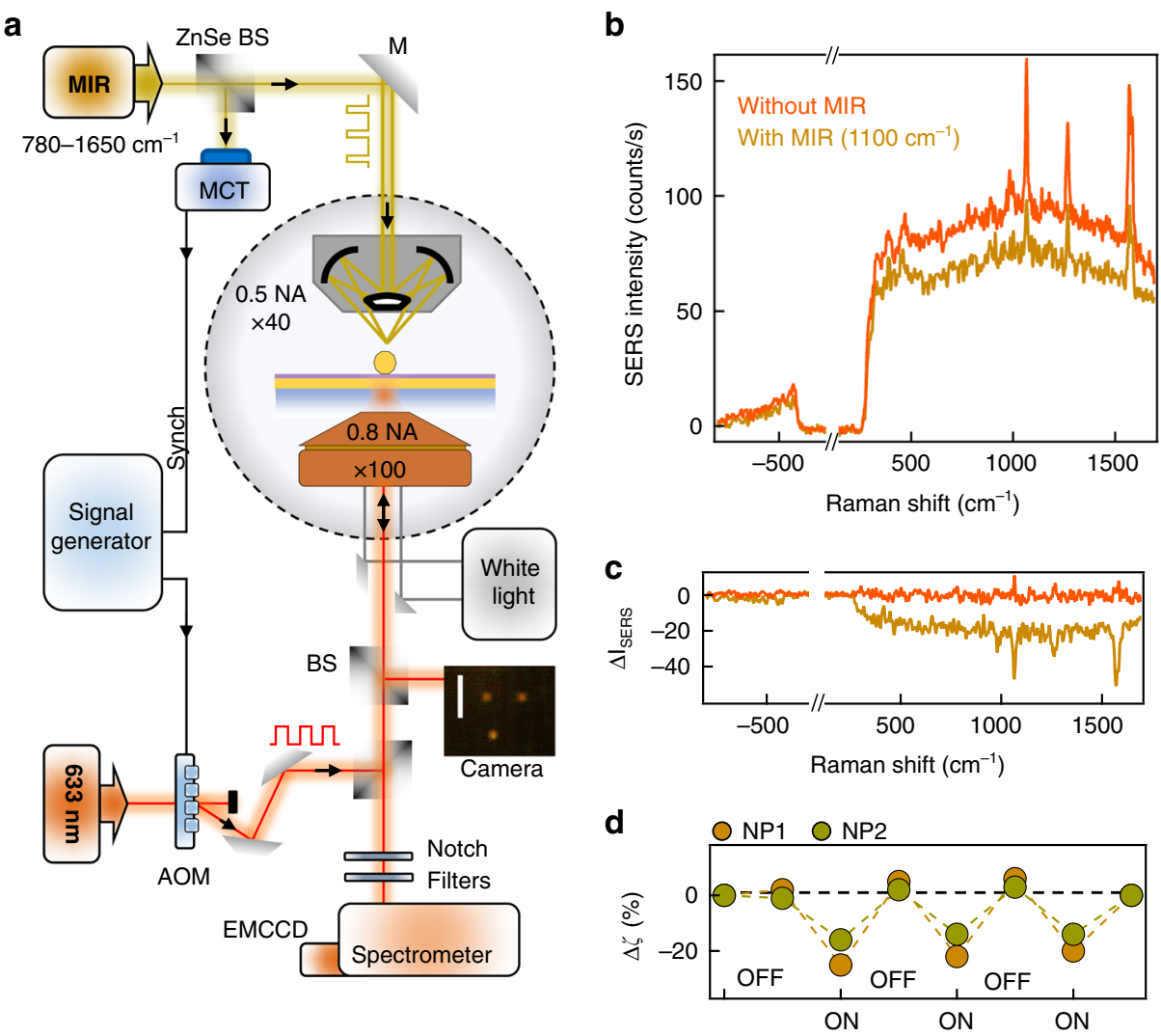

Fig. 2 MIR pump and visible SERS probe. a Setup of MIR (800-1600 $\left.\mathrm{cm}^{-1}\right)$ pump and visible $(633 \mathrm{~nm})$ probe beams illuminating an individual NPOF sample highlighted in black dotted circle. Acousto-optic modulator (AOM) for $633 \mathrm{~nm}$ SERS probe beam is synchronized with MIR pulses. SERS from NPoF sample is collected by a $0.8 \mathrm{NA} \times 100$ objective after filtering out probe laser with two notch filters. $\mathbf{b}$ SERS spectra from BPT selfassembled monolayer with and without the MIR pump at $1100 \mathrm{~cm}^{-1}$. c Difference in the SERS amplitude induced by MIR, averaged over 3 scans on a single NPoF. $\mathbf{d}$ Perturbed change $(\triangle \zeta \%)$ in SERS intensity (measured at $1100 \mathrm{~cm}^{-1}$ ) obtained by repeatedly switching the MIR beam ON/OFF for two different NPoFs 

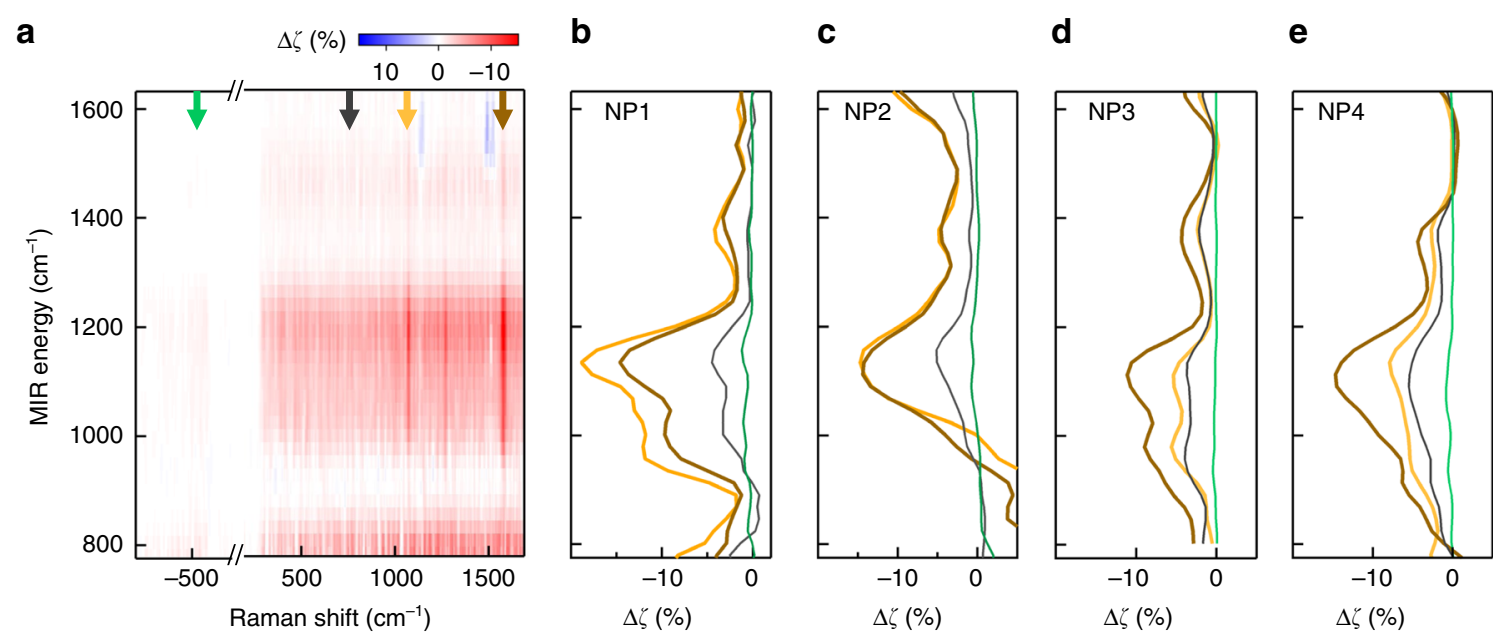

Fig. 3 MIR energy-dependent SERS change. a Perturbed $\Delta \zeta(\%)$ in the SERS signal from an individual NPoF when scanning the MIR frequency. $\Delta \zeta$ is normalized by the reference SERS before and after MIR illumination from the same NPoF cavity. b-e Perturbed $\triangle \zeta$ (\%) extracted at the BPT vibrational lines at: $1580 \mathrm{~cm}^{-1}$ (yellow), $1080 \mathrm{~cm}^{-1}$ (brown), Stokes electronic Raman scattering ERS (grey) and anti-Stokes ERS (green), indicated by arrows in (a). Analysis from three other NPoFs is shown in (c-e). (Unprocessed spectra in Supporting Information, Fig. S3, S4)

spectra over a period of $30 \mathrm{~s}$ from an individual NPoF cavity is $<1 \%$ (Supporting Information Fig. S2). Upon irradiating with MIR light at $1100 \mathrm{~cm}^{-1}$, the SERS intensity is found to decrease by $\Delta \zeta>20 \%$ (Fig. 2b). This strong decrease in SERS signal is observed across all the vibrational lines of BPT as well as the Stokes background from the electronic Raman scattering (Fig. 2c). The observed intensity change immediately recovers once the MIR light is turned off (Fig. 2d).

To understand the MIR energy dependence we collect SERS spectra while tuning the MIR energy between 800 and $1600 \mathrm{~cm}^{-1}$ (in steps of $20 \mathrm{~cm}^{-1}$ ). SERS spectra are also collected both before and after the sample is illuminated with MIR light for reference. Scans with large variations (> 30\%) in the SERS spectra before and after MIR illumination either due to the alignment drift or diffusion of Au-adatoms in NPoF gaps are not considered ${ }^{38-40}$. Perturbed changes $\Delta \zeta$ in SERS Stokes and anti-Stokes signals upon tuning the MIR illumination energy (Fig. 3a) show a broadband response. Line profiles extracted from BPT at two different vibrational lines $\left(1080 \mathrm{~cm}^{-1}\right.$ and $1585 \mathrm{~cm}^{-1}$, Fig. $3 \mathrm{~b}-\mathrm{e}$ ) show the maximum decrease occurs when the MIR is tuned around $1100 \mathrm{~cm}^{-1}$. Electronic Raman signals extracted from the Stokes background exhibit similar line profiles, with a lower magnitude (grey). This characteristic MIR-perturbed peak at $1100 \mathrm{~cm}^{-1}$ corresponds to the $\mathrm{SiO}_{2}$ phonon absorption (see below).

It is important to note that we never observe an increase in SERS, even when using AuNPs with different lower facet size which tunes their plasmon resonances with respect to the $633 \mathrm{~nm}$ probe wavelength (Supporting Information, Fig. S10). The measured scattering resonances match our simulations for AuNPs with average facet sizes of $20 \mathrm{~nm}$, which is consistent with scanning electron microscope images (Supporting Information, Fig. S9). In addition, experiments performed on the foil away from the AuNP do not show any signature of MIRperturbed SERS, which confirms that the perturbed SERS signals are observed only from the nanogap, and not from the foil on its own.

To confirm the origin of MIR-perturbed signals from the influence of vibrations of the support material underneath the foil, we constructed NPoF systems with polystyrene replacing $\mathrm{SiO}_{2}$ under the foil (Fig. 4a). The MIR energy dependence on polystyrene-NPoFs show a different spectral dependence and weaker signal intensity compared to $\mathrm{SiO}_{2}$ samples. Here a smaller MIR frequency range is scanned with a finer resolution of $5 \mathrm{~cm}^{-1}$. The MIR-perturbed SERS spectrum displays sharp peaks matching the vibrational absorption lines of bulk polystyrene, clearly indicating that the signal must originate from interactions with the material underneath the foil. The perturbed SERS signal varies across different NPoF structures which is a characteristic signature of nanoscale inhomogeneities, depending on the exact molecular geometry of polymer underneath the NPoF.

To characterize the dynamics of the MIR-perturbed SERS signal originating from the phonons underneath the foil, we develop a time-correlated single-photon lock-in method to time-resolve the signal. The Stokes part of the SERS signal is routed to a single-photon avalanche diode (SPAD) detector (Fig. 5a). The arrival of each SERS photon is time-correlated to the MIR trigger signal from the quantum cascade laser (QCL). This allows us to resolve the MIR-perturbed signal with a time resolution of 

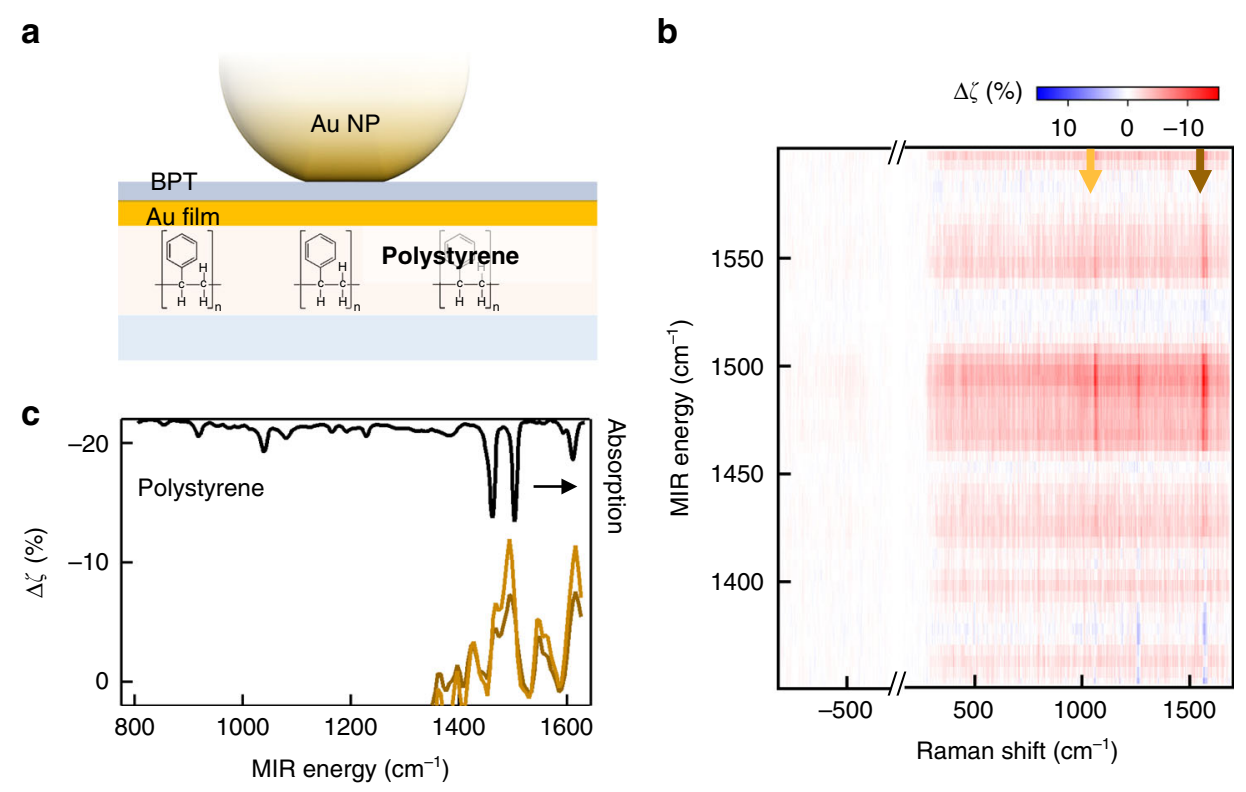

Fig. 4 MIR-perturbed SERS for polystyrene NPoF. a NPoF sample with 500-nm-thick polystyrene underneath the Au-foil. b MIR perturbed $\triangle \zeta$ (\%) in the SERS signal from an individual polystyrene NPoF when scanning the MIR frequency (unprocessed spectra in Supporting Information, Fig. S13a). c $\Delta \zeta(\%)$ extracted at the BPT vibrational lines at: $1580 \mathrm{~cm}^{-1}$ (yellow) and $1080 \mathrm{~cm}^{-1}$ (brown) from (b) and compared with the bulk polystyrene absorption (black)

$100 \mathrm{ps}^{41}$. The QCL is triggered at $0.32 \mathrm{MHz}$ with MIR pulses of width $100 \mathrm{~ns}$. The MIR-perturbed SERS rapidly decreases immediately after the MIR pulse (Fig. 5b), with a timescale of $\sim 300 \mathrm{~ns}$ consistently obtained across multiple NPoF cavities (not limited by the MIR pulsewidth of $100 \mathrm{~ns})$. Subsequently, the SERS signal recovers with a longer decay time of $>500 \mathrm{~ns}$. This temporal response is fit with the single exponential rise and decay times using experiments on $>25 \mathrm{NPoF}$ cavities. The rise time is narrowly distributed around $290 \pm 50 \mathrm{~ns}$ whereas the decay time is more variable spanning $700 \pm 250 \mathrm{~ns}$ (Fig. 5c). We find a positive correlation between the rise and decay times $\left(\tau_{\text {decay }} \sim 3.9 \tau_{\text {rise }}\right)$, suggesting they are intrinsically linked to the origin of the MIR-perturbed SERS signature.

\section{Discussion}

The maximum decrease in SERS signal observed at MIR energies $\sim 1100 \mathrm{~cm}^{-1}$ is consistent across different NPoFs; however, the magnitude of signal varies between $\Delta \zeta=$ $10-25 \%$. The spectral response of the perturbed SERS does not match with Raman or IR vibrational lines of BPT. This indicates that MIR absorption in BPT is not the dominant contribution to the observed signal. Instead, this characteristic peak at $1100 \mathrm{~cm}^{-1}$ corresponds to the $\mathrm{SiO}_{2}$ phonon absorption. This is also evidenced in the reflection dip that exhibits a typical Reststrahlen band ${ }^{42,43}$ and confirmed by simulations of the MIR absorption at the $\mathrm{Au}-\mathrm{SiO}_{2}$ interface (Fig. 6a, b). Within a band between 900 and $1150 \mathrm{~cm}^{-1}$, the real part of the $\mathrm{SiO}_{2}$ dielectric function is negative $(\operatorname{Re}(\varepsilon)<0)$. The reduced SERS signal must therefore arise from a decrease in near-field intensity of the $633 \mathrm{~nm}$ probe, somehow caused by MIR excitation of this confined mode at the $\mathrm{Au}-\mathrm{SiO}_{2}$ interface. This results in a linear response with MIR power (Fig. 6c).

Direct heating of the $\mathrm{Au}$ interface from the $2 \mathrm{~mW}$ average power MIR pump contributes only a minimal change of $<1{ }^{\circ} \mathrm{C}$ in temperature (Fig. $6 \mathrm{~d}$, e), which is fully consistent with the unchanged anti-Stokes background of SERS signals observed. The change in refractive index of $\mathrm{SiO}_{2}$ needed to account for a $20 \%$ decrease in SERS is rather high $(\Delta n>0.2)$ for the pump powers used here (Supporting Information, Fig. S5), corresponding to temperatures $>1000^{\circ} \mathrm{C}$. As a result, simple thermal effects are not sufficient to account for these observations. Further, conventional photothermal signals possess slow timescales $(\mathrm{ms})^{44,45}$ as the induced deflection of visible light requires strong deformations of the substrate underneath. Thermal expansion of $\mathrm{Au}$ or $\mathrm{SiO}_{2}$ for a $10 \mathrm{~K}$ increase in local temperature is far too small to modulate the visible probe as required (Supporting Information, Table. S1). Similarly, reversible reconstruction of grain boundaries or polycrystallinity in the $\mathrm{Au}$-foil on $\mathrm{SiO}_{2}$ seems also unlikely to explain this MIR-perturbed SERS.

The decrease in the SERS signal is thus attributed to a shift in the plasmon resonance wavelength perturbed by the modulation of refractive index directly around the AuNP (Supporting Information, Fig. S6). Exciting the $\mathrm{SiO}_{2}$ Reststrahlen band shifts the (10) NPoF plasmon by 

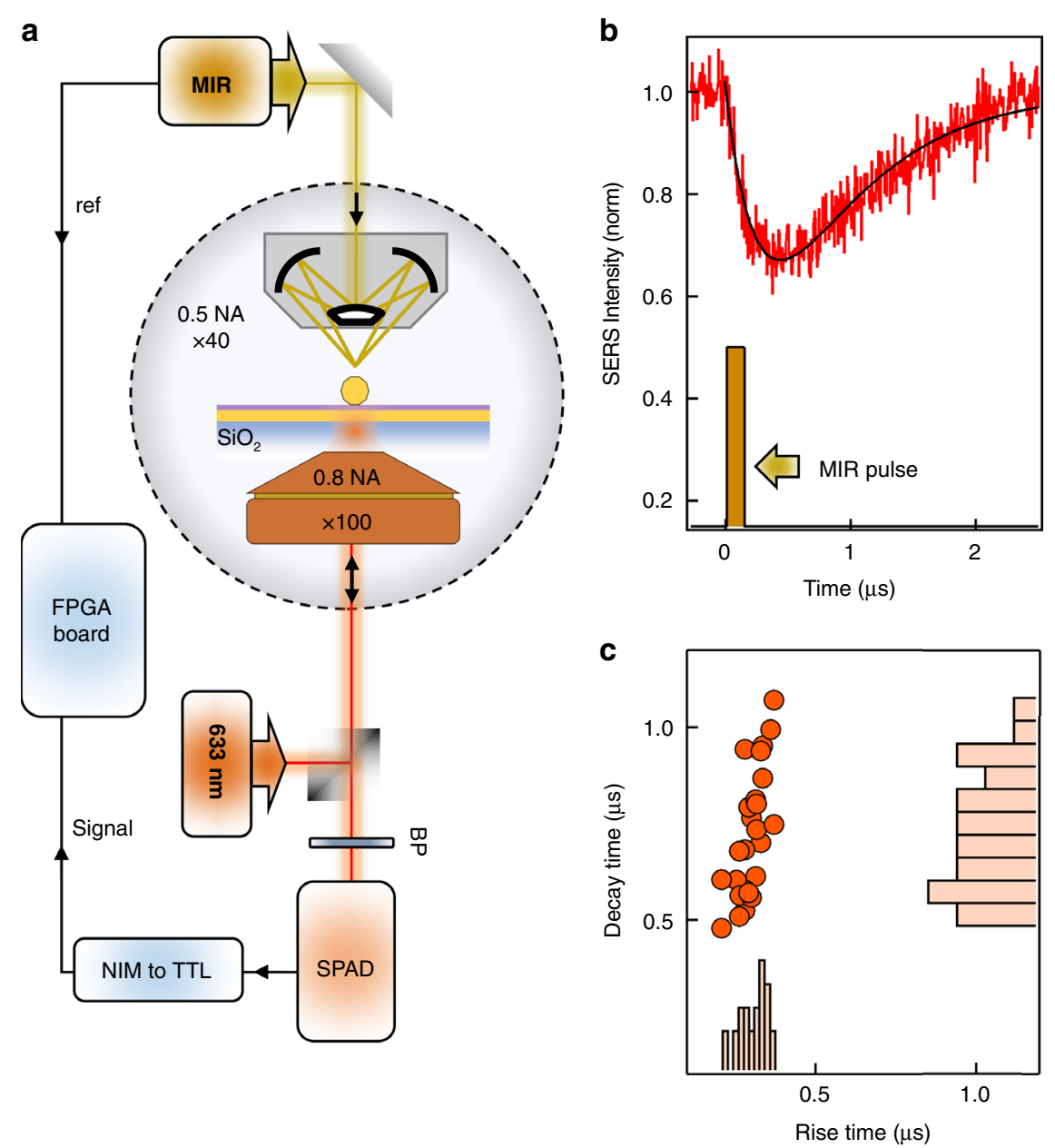

Fig. 5 Time-resolved single-photon lock-in measurements. a Modified microscope correlates trigger signals from MIR laser with SERS photons (filtered by band-pass BP) detected by single-photon avalanche diode (SPAD) via custom field-programmable gate array (FPGA) board. Single-photon detection by the SPAD is read out through a signal converter (NIM to TTL) onto the FPGA board. b Time-resolved MIR-perturbed SERS signal (red) from 100 ns MIR pulse. Black curve is fit to extract rise and decay times. c Variation in rise time correlates with decay time, histograms of rise, decay times plotted on corresponding axes

$\sim 1 \mathrm{~nm}$ and reduces the plasmon enhancement of SERS at $\lambda=633 \mathrm{~nm}$. We can identify very few possible routes for this modulation, but possibilities can be either from nmscale deformations in the NP surface or from the effects of a nanoscale shell of water in the crevices under the AuNP. This shell of water is always present for such nanoassemblies in ambient conditions, and extremely hard to remove due to the highly acute crevice angle. The experiments performed here are in ambient dry conditions. However, in such nanogap confined environments, trapped water rearranges into various phases and can never be driven off completely ${ }^{46}$. We perform additional experiments with NPoF samples immersed in water and ethanol where evaporation is absent, and this indeed gives undetectable perturbation of the SERS signal in the presence of MIR light (Supporting Information, Fig. S8). Modelling shows that changing the crevice water shell width by $<5 \mathrm{~nm}$ is sufficient to induce a $20 \%$ decrease in the SERS signal (Fig. 6f, g). While the weak direct absorption of MIR light is insufficient to induce this, the situation is very different in the spectral band where $\mathrm{SiO}_{2}$ acts as a metal ${ }^{47}\left(\operatorname{Re}(\varepsilon)<0\right.$ from 900 to $\left.1200 \mathrm{~cm}^{-1}\right)$ which allows it to support surface-plasmon-polaritons (SPPs) that amplify the optical field near Au by $>50$. These MIR SPPs are excited only by scattering at the NP, leading to even higher fields directly in the crevices and thus heating trapped water in real time. Indeed, replacing the $\mathrm{SiO}_{2}$ with $\mathrm{Si}_{3} \mathrm{~N}_{4}$ (which has $\operatorname{Re}(\varepsilon)>0$ throughout our spectral range) eliminates this effect, demonstrating the key role of resonant MIR SPPs ${ }^{22}$. The total absorbed energy from each MIR pulse is a hundred-fold larger than required to evaporate a 5 $\mathrm{nm}$ shell of water. This mechanism is also consistent with the sub- $\mu$ s rise and decay times, which correspond to thermal diffusion times from the heated nanoparticle (Fig. 6f). 


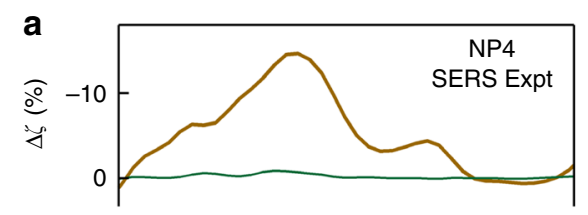

b
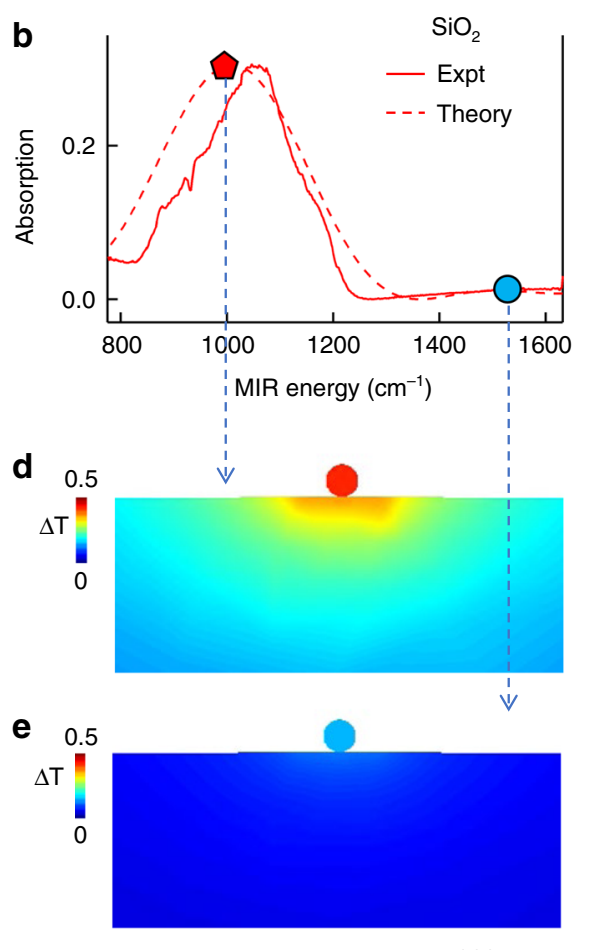

$200 \mathrm{~nm}$

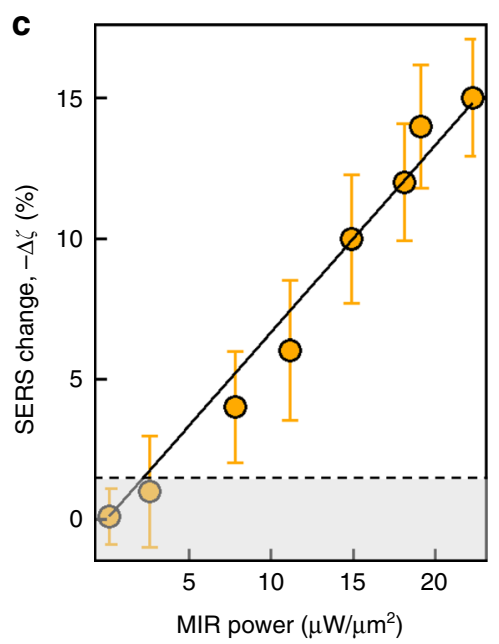

$\mathbf{f}$
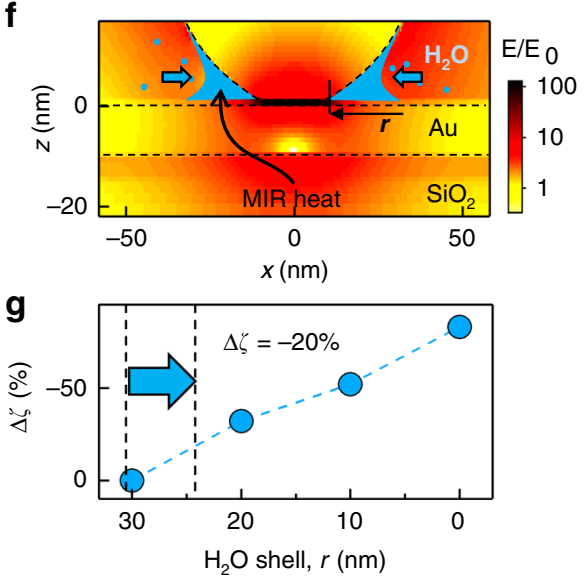

Fig. 6 SERS attenuation due to $\mathrm{SiO}_{2}$ phonon absorption. a Perturbed $\Delta \zeta(\%)$ in the SERS signal from an individual NPoF when scanning the MIR frequency. $\mathbf{b}$ Experimental and FDTD-simulated MIR absorption spectra of $\mathrm{SiO}_{2}$-Au interface obtained in reflection geometry. $\mathbf{c ~ M I R ~}\left(1100 \mathrm{~cm}{ }^{-1}\right)$ power-dependent variation in the $\Delta \zeta(\%)$ of SERS signal. Solid line is linear fit, dashed gives detection noise baseline. $\mathbf{d}$, e Steady-state heat profile in NPoF simulated at (d) $1000 \mathrm{~cm}^{-1}$ and (e) $1500 \mathrm{~cm}^{-1}$. f Near-field enhancement of NPoF at $705 \mathrm{~nm}\left(1580 \mathrm{~cm}^{-1}\right.$ SERS line) surrounded by a $30-\mathrm{nm}$ thick water shell (blue). $\mathbf{g}$ Simulated $\Delta \zeta(\%)$ for decreasing water shell thicknesses

Most dielectrics support vibrational mid-infrared-active phonon modes which interact with light and plasmons in the same fashion as described above ${ }^{48,49}$. These polariton modes are distributed across the MIR-visible regions and constrain the nanoscale geometries for producing upconverted SERS signals. Since MIR SPP excitation improves the MIR coupling into the gap, there is a tradeoff between enhanced SERS upconversion and enhanced thermally perturbed retuning of the plasmon resonances. Our work suggests that avoiding the substrate Reststrahlen band will be needed for observing SERS upconversion from molecules ${ }^{22}$.

The efficiencies of MIR detection in this NPoF system are compared with state-of-the-art low-dimensional semiconductor heterostructures or graphene that have been implemented for $\mathrm{THz}$ detection in recent detection schemes $^{50-53}$. From an application perspective, the relevant figure of merit is the noise equivalent power (NEP), which corresponds to the lowest detectable power in $0.5 \mathrm{~s}$ integration time. This is measured here as the MIR power-dependent perturbation to the SERS signal (Fig. 6c); however, most of the incident MIR is reflected by the $\mathrm{Au}$-foil and remains undetected instead of being absorbed in the substrate. Given the $100 \mathrm{~nm}^{2}$ crosssection of NPoFs at MIR frequencies, the NEP is estimated to be $0.1 \mathrm{nW} \mathrm{Hz}{ }^{-0.5}$, which is close to state-of-theart detectors. Carefully designed variants of the NPoF geometry with MIR antenna resonances supporting unity absorption of MIR light would greatly boost the NEP. Theoretically the noise level is limited by photon shot noise in the visible laser, although in current experiments the noise is limited by the stability of the SERS signal. Light- 
driven diffusion of adatoms $\mathrm{s}^{38,54}$ and fluctuations ${ }^{39}$ of defects in the metal nanoparticle contribute to significant variation in SERS intensities. There exists an opportunity to significantly improve the noise reduction by developing more robust nanocavity systems. Further, we suggest additional improvements in MIR detection by deterministically creating adatom picocavities with light ${ }^{40,55}$.

In summary, we show how molecular SERS signals are modified by irradiating with MIR light across a wide spectral bandwidth from 5.8 to $12 \mu \mathrm{m}(24-51 \mathrm{THz}$, $800-1700 \mathrm{~cm}^{-1}$ ). Our observations reveal that phonon resonances of the $\mathrm{SiO}_{2}$ substrate trap intense MIR SPPs in the Reststrahlen band, which can temporarily retune the localized plasmons by perturbing the outer 5 -nm-thick shells of water in the nanostructure crevices. This results in strong reductions in SERS intensity, but could also be used in other ways, for instance for tuning plasmons in real time, as well as for exciting the NPoM in the MIR through SPP waveguides or antenna coupling. This suggests new ways to access nanoscale chemical imaging ${ }^{3}$, MIR photothermal bolometers ${ }^{56}$, photoacoustic microscopy $^{57}$ and optomechanics ${ }^{58}$.

\section{Materials and methods}

\section{Sample preparation}

To prepare the thin mirror, we deposit $10 \mathrm{~nm}$ of Au on a clean $\mathrm{SiO}_{2}$ cover slip $(150 \mu \mathrm{m}$ thick) with a deposition rate of $0.5 \AA \mathrm{s}^{-1}$ (Moorfield nanoPVD-T15A thermal evaporator). The $\mathrm{Au}$-coated $\mathrm{SiO}_{2}$ substrates are dipped into a $1 \mathrm{mM}$ solution of biphenyl-4-thiol (BPT, Sigma Aldrich, 97\%) in anhydrous ethanol (Sigma Aldrich, $<0.003 \%$ H2O) for $12 \mathrm{~h}$ resulting in self-assembled molecular monolayers (SAMs). For NPoF optical cavities, $80 \mathrm{~nm}$ faceted NPs (BBI Solutions) are deposited directly onto the BPT-assembled Au-coated $\mathrm{SiO}_{2}$ substrates. The deposition time is kept below $30 \mathrm{~s}$, resulting in well-dispersed NPs. Lastly, the samples are rinsed thoroughly with double distilled water to remove the excess AuNPs.

\section{Experimental setup}

All SERS and MIR spectroscopy measurements are performed in a custom-built dual-channel microscope. For SERS, a spectrally filtered $633 \mathrm{~nm}$ diode laser

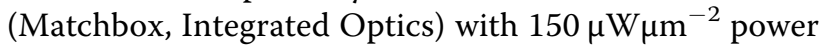
on the sample is used as a probe and is filtered with two notch filters before routing it to a Shamrock i303 spectrograph and a Newton EMCCD. The $633 \mathrm{~nm}$ light is focused onto the sample with the aid of a $\times 100$ 0.8 NA long working distance microscope objective. For imaging, the reflected light collected through the same objective lens is directed to a camera (Lumenera Infinity3-1). For the MIR light source, a quantum cascade laser (QCL) from LaserTune IR source (Block) with wavelength range of $5.4-13 \mu \mathrm{m}$ is used $\left(1635-780 \mathrm{~cm}^{-1}\right)$ and maximum average output of $500 \mu \mathrm{W}(\sim 2 \times 4 \mathrm{~mm}$ collimated) with $5 \%$ duty cycle. The pump (MIR light) is coaligned with the probe (visible light) using a $0.4 \mathrm{NA}$ Cassegrain objective lens. For MIR detection, an external mercury-cadmium-telluride (MCT) IR detector is used along with a $\mathrm{ZnSe}$ beam-splitter and is synced with the AOM to modulate the $633 \mathrm{~nm}$ diode laser. This improves the pump and probe pulse temporal overlap by matching the repetition rate and pulse widths. The sample is placed on a fully automated motorized stage (Prior Scientific H101) which is controlled with code written in Python.

For single-photon time-correlated measurements, arrival times of all photons at the detector (Micro Photon Devices PDM \$PD-100-CTD) and reference signals (MIR laser trigger) are continuously recorded by a time-todigital converter on a field-programmable gate array (FPGA) board. Comparing the photon timestamps with the reference signal allows recreating the periodic perturbation of the SERS signal by the MIR laser in time, integrated over millions of modulation cycles. This singlephoton lock-in detection scheme is described in more detail elsewhere ${ }^{41}$.

\section{Acknowledgements \\ The authors acknowledge support from European Research Council (ERC) under Horizon 2020 research and innovation programme PICOFORCE (Grant Agreement No. 861950), THOR (Grant Agreement No. 829067) and POSEIDON (Grant Agreement No. 861950). We acknowledge funding from the EPSRC (Cambridge NanoDTC EP/L015978/1, EP/L027151/1, EP/S022953/1, EP/ P029426/1, and EP/R020965/1). R.C. acknowledges support from Trinity College, University of Cambridge.}

\section{Author contributions}

R.C and J.J.B conceived and designed the experiments. R.C. performed the experiments with input from A.X and L.A.J. R.C. carried out the simulation and the analytical modelling with input from A.X. R.C., A.X. and J.J.B. analysed the data. R.C. and J.J.B. wrote the manuscript with input from all authors.

\section{Data availability}

Source data can be found at https://doi.org/10.17863/CAM.79290.

Conflict of interest

The authors declare no competing interests.

Supplementary information The online version contains supplementary material available at https://doi.org/10.1038/s41377-022-00709-8.

Received: 12 July 2021 Revised: 15 November 2021 Accepted: 5 January 2022

Published online: 19 January 2022

\footnotetext{
References

1. Cheng, J. X. \& Xie, X. S. Vibrational spectroscopic imaging of living systems: an emerging platform for biology and medicine. Science 350, 6264 (2015).

2. Huth, F. et al. Infrared-spectroscopic nanoimaging with a thermal source. Nat. Mater. 10, 352-356 (2011).

3. O'Callahan, B. T. et al. Photoinduced tip-sample forces for chemical nanoimaging and spectroscopy. Nano Lett. 18, 5499-5505 (2018).

4. Low, T. et al. Polaritons in layered two-dimensional materials. Nat. Mater. 16, 182-194 (2017).
} 
5. Song, B. et al. Enhancement of near-field radiative heat transfer using polar dielectric thin films. Nat. Nanotechnol. 10, 253-258 (2015).

6. Basov, D. N., Fogler, M. M. \& de Abajo, J. F. G. Polaritons in van der Waals materials. Science 354, aag1992 (2016).

7. Dorling, K. M. \& Baker, M. J. Rapid FTIR chemical imaging: highlighting FPA detectors. Trends Biotechnol. 31, 437-438 (2013).

8. Baker, M. J. et al. Using Fourier transform IR spectroscopy to analyze biological materials. Nat. Protoc. 9, 1771-1791 (2014).

9. Dam, J. S., Tidemand-Lichtenberg, P. \& Pedersen, C. Room-temperature midinfrared single-photon spectral imaging. Nat. Photonics 6, 788-793 (2012).

10. Kviatkovsky, I. et al. Microscopy with undetected photons in the mid-infrared. Sci. Adv. 6, eabd0264 (2010).

11. Junaid, S. et al. Video-rate, mid-infrared hyperspectral upconversion imaging. Optica 6, 702-708 (2019).

12. Knez, D. et al. Infrared chemical imaging through non-degenerate two-photon absorption in silicon-based cameras. Light.: Sci. Appl. 9, 125 (2020).

13. Kutas, M. et al. Quantum-inspired terahertz spectroscopy with visible photons. Optica 8, 438-441 (2021).

14. Lindner, C. et al. Nonlinear interferometer for Fourier-transform mid-infrared gas spectroscopy using near-infrared detection. Opt. Express 29, 4035-4047 (2021).

15. Tidemand-Lichtenberg, P. et al. Mid-infrared upconversion spectroscopy. J. Opt. Soc. Am. B 33, D28-D35 (2016).

16. Wang, L. et al. Nanoscale simultaneous chemical and mechanical imaging via peak force infrared microscopy. Sci. Adv. 3, e1700255 (2017).

17. Anderson, M. S. Infrared spectroscopy with an atomic force microscope. Appl. Spectrosc. 54, 349-352 (2000).

18. Bai, Y. R., Yin, J. Z. \& Cheng, J. X. Bond-selective imaging by optically sensing the mid-infrared photothermal effect. Sci. Adv. 7, eabg1559 (2021).

19. Bagci, $T$. et al. Optical detection of radio waves through a nanomechanical transducer. Nature 507, 81-85 (2014).

20. Belacel, $C$. et al. Optomechanical terahertz detection with single meta-atom resonator. Nat. Commun. 8, 1578 (2017).

21. Roelli, P. et al. Molecular platform for frequency upconversion at the singlephoton level. Phys. Rev. X 10, 031057 (2020).

22. Xomalis, A. et al. Detecting mid-infrared light by molecular frequency upconversion with dual-wavelength hybrid nanoantennas. Science 374, 1268 (2021).

23. Zhang, Y., Aizpurua, J. \& Esteban, R. Optomechanical collective effects in surface-enhanced Raman scattering from many molecules. ACS Photonics 7, 1676-1688 (2020).

24. Neuman, T. et al. Quantum description of surface-enhanced resonant Raman scattering within a hybrid-optomechanical model. Phys. Rev. A 100, 043422 (2019).

25. Rodrigo, D. et al. Self-similar multiresonant nanoantenna arrays for sensing from near- to mid-infrared. ACS Photonics 5, 4903-4911 (2018).

26. Le, F. et al. Metallic nanoparticle arrays: a common substrate for both surfaceenhanced Raman scattering and surface-enhanced infrared absorption. ACS Nano 2, 707-718 (2008).

27. Neubrech, F. et al. Surface-enhanced infrared spectroscopy using resonant nanoantennas. Chem. Rev. 117, 5110-5145 (2017).

28. Huh, J. H., Lee, J. \& Lee, S. Soft plasmonic assemblies exhibiting unnaturally high refractive index. Nano Lett. 20, 4768-4774 (2020).

29. Schuller, J. A. et al. Plasmonics for extreme light concentration and manipulation. Nat. Mater. 9, 193-204 (2010).

30. $\mathrm{Xu}, \mathrm{Y}$. et al. Light-matter interaction within extreme dimensions: from nanomanufacturing to applications. Adv. Opt. Mater. 6, 1800444 (2018).

31. Gramotnev, D. K. \& Bozhevolnyi, S. I. Nanofocusing of electromagnetic radiation. Nat. Photonics 8, 13-22 (2014).
32. Urbieta, M. et al. Atomic-scale lightning rod effect in plasmonic picocavities: a classical view to a quantum effect. ACS Nano 12, 585-595 (2018).

33. Chikkaraddy, R. et al. How ultranarrow gap symmetries control plasmonic nanocavity modes: from cubes to spheres in the nanoparticle-on-mirror. ACS Photonics 4, 469-475 (2017).

34. Baumberg, J. J. et al. Extreme nanophotonics from ultrathin metallic gaps. Nat. Mater. 18, 668-678 (2019).

35. Kongsuwan, $\mathrm{N}$. et al. Plasmonic nanocavity modes: from near-field to far-field radiation. ACS Photonics 7, 463-471 (2020).

36. Chikkaraddy, R. \& Baumberg, J. J. Accessing plasmonic hotspots using nanoparticle-on-foil constructs. ACS Photonics 8, 2811-2817 (2021).

37. Benz, F. et al. SERS of individual nanoparticles on a mirror: size does matter, but so does shape. J. Phys. Chem. Lett. 7, 2264-2269 (2016).

38. Xomalis, A. et al. Controlling optically driven atomic migration using crystalfacet control in plasmonic nanocavities. ACS Nano 14, 10562-10568 (2020).

39. Carnegie, C. et al. Flickering nanometre-scale disorder in a crystal lattice tracked by plasmonic flare light emission. Nat. Commun. 11, 682 (2020).

40. Carnegie, C. et al. Room-temperature optical picocavities below $1 \mathrm{~nm} 3$ accessing single-atom geometries. J. Phys. Chem. Lett. 9, 7146-7151 (2018).

41. Jakob, L. A. et al. Single-photon multiclock lock-in detection by picosecond timestamping. Optica 8, 1646 (2021).

42. Amarie, S. \& Keilmann, F. Broadband-infrared assessment of phonon resonance in scattering-type near-field microscopy. Phys. Rev. B 83, 045404 (2011).

43. Li, P. N. et al. Reversible optical switching of highly confined phonon-polaritons with an ultrathin phase-change material. Nat. Mater. 15, 870-875 (2016).

44. Baffou, G. et al. Simple experimental procedures to distinguish photothermal from hot-carrier processes in plasmonics. Light.: Sci. Appl. 9, 108 (2020).

45. Aleshire, K. et al. Far-field midinfrared superresolution imaging and spectroscopy of single high aspect ratio gold nanowires. Proc. Natl Acad. Sci. USA 117, 2288-2293 (2020).

46. Asay, D. B. \& Kim, S. H. Effects of adsorbed water layer structure on adhesion force of silicon oxide nanoasperity contact in humid ambient. J. Chem. Phys. 124, 174712 (2006)

47. Turner, A. F., Chang, L. \& Martin, T. P. Enhanced reflectance of reststrahlen reflection filters. Appl. Opt. 4, 927-933 (1965).

48. Freitag, M. et al. Substrate-sensitive mid-infrared photoresponse in graphene. ACS Nano 8, 8350-8356 (2014).

49. Autore, $\mathbf{M}$. et al. Substrate matters: surface-polariton enhanced infrared nanospectroscopy of molecular vibrations. Nano Lett. 19, 8066-8073 (2019).

50. Alves, F. et al. Microelectromechanical systems bimaterial terahertz sensor with integrated metamaterial absorber. Opt. Lett. 37, 1886-1888 (2012).

51. Todorov, Y. et al. Ultrastrong light-matter coupling regime with polariton dots. Phys. Rev. Lett. 105, 196402 (2010).

52. Vicarelli, L. et al. Graphene field-effect transistors as room-temperature terahertz detectors. Nat. Mater. 11, 865-871 (2012).

53. Scalari, G. et al. Ultrastrong coupling of the cyclotron transition of a $2 \mathrm{D}$ electron gas to a THz metamaterial. Science 335, 1323-1326 (2012).

54. Chikkaraddy, R. et al. Dynamics of deterministically positioned single-bond surface-enhanced Raman scattering from DNA origami assembled in plasmonic nanogaps. J. Raman Spectrosc. 52, 348-354 (2021).

55. Benz, F. et al. Single-molecule optomechanics in "picocavities". Science $\mathbf{3 5 4}$ 726-729 (2016).

56. Efetov, D. K. et al. Fast thermal relaxation in cavity-coupled graphene bolometers with a Johnson noise read-out. Nat. Nanotechnol. 13, 797-801 (2018).

57. Shi, J. H. et al. High-resolution, high-contrast mid-infrared imaging of fresh biological samples with ultraviolet-localized photoacoustic microscopy. Nat. Photonics 13, 609-615 (2019).

58. Shishkov, V. Y. et al. Enhancement of the Raman effect by infrared pumping. Phys. Rev. Lett. 122, 153905 (2019). 